\title{
Pressure-assisted photopolymerization in the molecular donor-acceptor fullerene complex $\left\{\mathrm{Cd}(\text { dedtc })_{2}\right\}_{2} \cdot \mathrm{C}_{60}$
}

\author{
K. P. Meletov ${ }^{1 *}$, J. Arvanitidis ${ }^{2}$, D. Christofilos ${ }^{2}$, G. Kourouklis ${ }^{2}$ \\ ${ }^{1}$ Institute of Solid State Physics, Russian Academy of Sciences, Chernogolovka, \\ Moscow region, Russia \\ ${ }^{2}$ Aristotle University of Thessaloniki, GR-54 124 Thessaloniki, Greece \\ *mele@issp.ac.ru
}

PACS 78.30.Na

DOI 10.17586/2220-8054-2016-7-1-133-139

\begin{abstract}
The pressure-assisted photopolymerization in the fullerene complex $\left\{\mathrm{Cd}(\mathrm{dedtc})_{2}\right\}_{2} \cdot \mathrm{C}_{60}$ (fullerene complex with cadmium diethyldithiocarbamate) observed in the pressure region between 2.24 and 5.99 GPa was studied in detail. After a phase transition near $\sim 2 \mathrm{GPa}$, the Raman spectra exhibit time-dependent changes under prolonged laser illumination regarding the appearance of new peaks in the frequency region of the $\mathrm{A}_{g}(2)$ mode of the $\mathrm{C}_{60}$ monomer. These peaks are related to the fullerene oligomers with a different number of $s p^{3}$-like coordinated carbon atoms per molecular cage. The polymer content increases with the laser power density and exposure time, while for a fixed laser power, its saturated value is independent of pressure. Outside the pressure range 2.24 - 5.99 GPa, photopolymerization is suppressed.
\end{abstract}

Keywords: high pressure, fullerene, molecular complexes, Raman scattering, photopolymerization.

Received: 20 November 2015

\section{Introduction}

The high pressure behavior of donor-acceptor fullerene complexes is of interest due to their layered structure in which the close-packed fullerene layers are alternated with layers of the molecular donor. At high pressures, the reduction of intermolecular distances in fullerene complexes increases the overlap of the highest occupied molecular orbital (HOMO) of the molecular donor and the lowest unoccupied molecular orbital (LUMO) of the fullerene acceptor. This may cause charge transfer, particularly in the case where the molecular complex at ambient conditions is close to the ionic state. Alternatively, the decrease of intermolecular distances within the fullerene layers may result in the formation of covalent inter-cage bonds. The $X$-ray diffraction (XRD) and Raman studies of ionic and neutral donor-acceptor complexes of $\mathrm{C}_{60}$ have revealed the formation of covalent bonds between fullerene molecules at low temperature or elevated pressure [1-8]. Recently, the photo-induced transformations at high pressure were observed in the Raman spectra of the molecular complex $\left\{\mathrm{Cd}(\mathrm{dedtc})_{2}\right\}_{2} \cdot \mathrm{C}_{60}$ which take place immediately after the pressure-induced phase transition near $2 \mathrm{GPa}$ [9]. The time-dependent changes in the $\mathrm{A}_{g}(2)$ mode region are reminiscent of those in the Raman spectra of $\mathrm{C}_{60}$ fullerene under light irradiation [10].

The ability of $\mathrm{C}_{60}$ to form inter-cage covalent bonds is due to the existence of 30 unsaturated double $\mathrm{C}=\mathrm{C}$ bonds in the $\mathrm{C}_{60}$ molecule. $\mathrm{C}_{60}$ fullerite polymerizes under illumination [10], alkali metal doping [11] and high-pressure/high-temperature (HPHT) treatment [12,13]. The polymerization of $\mathrm{C}_{60}$ is realized through the creation of covalent bonds among adjacent fullerene molecules via a [2+2] cyclo-addition mechanism that necessitates the rotation of molecules to attain a suitable mutual orientation $[10,14]$. The photopolymerization is efficient in thin films or the surfaces of bulk samples, owing to the small light penetration depth 
( $\sim 1 \mu \mathrm{m}$ near $500 \mathrm{~nm}$ ); the photo-transformed material is rather disordered and contains various oligomers $[15,16]$. For these reasons, the XRD method is unsuitable for the analysis of the photopolymers and the role of the Raman spectroscopy becomes crucial. Unlike the case of the photopolymers, $\mathrm{XRD}$ is very useful in the study of the ordered crystalline $\mathrm{C}_{60}$ polymers obtained by HPHT treatment of the $\mathrm{C}_{60}$ fullerite whose structures have been identified as one-dimensional orthorhombic (1D-O), two-dimensional tetragonal (2D-T) and two-dimensional rhombohedral (2D-R) [12,13]. The Raman spectra of the crystalline polymers exhibit splitting and softening of the bands related to the lowering of the $\mathrm{C}_{60}$ molecular symmetry caused by the intermolecular covalent bonds $[12,13,17]$. An important probe of the $\mathrm{C}_{60}$ polymers is the behavior of the $\mathrm{A}_{g}(2)$ pentagonal pinch (PP) mode of the $\mathrm{C}_{60}$ molecule, related to the in-phase stretching vibration that involves tangential displacements of carbon atoms with contraction of the pentagonal rings and expansion of the hexagonal rings. The PP-mode frequency is downshifted due to the lowering of the average cage stiffness with an increase in the number of the $s p^{3}$-like coordinated carbon atoms per $\mathrm{C}_{60}$ molecule, associated with the inter-cage bonds. Thus, the $\mathrm{A}_{g}(2)$ mode of the $\mathrm{C}_{60}$ monomer, observed at $1468 \mathrm{~cm}^{-1}$, shifts to $1458 \mathrm{~cm}^{-1}$ in the linear chains (1D-O polymer, $4 s p^{3}$-like coordinated carbon atoms), while in the planar polymers, the peak downshifts further to $1446 \mathrm{~cm}^{-1}$ (2D-T polymer, $8 s p^{3}$-like coordinated carbon atoms) and $1408 \mathrm{~cm}^{-1}$ (2D-R polymer, $12 s p^{3}$-like coordinated carbon atoms) [17].

In this paper, we present the detailed study of the pressure-assisted photopolymerization in the fullerene complex $\left\{\mathrm{Cd}(\mathrm{dedtc})_{2}\right\}_{2} \cdot \mathrm{C}_{60}$. We have carefully defined the pressure region in which the phototransformation takes place; we have studied the photopolymerization kinetics and its dependence on pressure, and identified the final products of the reaction as fullerene photo-oligomers. Starting at a pressure of $2.24 \mathrm{GPa}$, immediately after the pressure-induced phase transition [9], photopolymerization occurs up to $5.99 \mathrm{GPa}$, after which it is abruptly suppressed. In this pressure range, the Raman spectra exhibit time-dependent changes associated with the appearance of new bands in the frequency region of the $\mathrm{A}_{g}(2)$ mode of the $\mathrm{C}_{60}$ monomer. Their intensity increases exponentially with the laser exposure time and saturates at a value that does not depend on pressure. The peak frequencies indicate that the new peaks are related to photo-oligomers with four and six $s p^{3}$-like coordinated carbon atoms per molecular cage.

\section{Experimental details}

Samples of the donor-acceptor complex $\left\{\mathrm{Cd}(\mathrm{dedtc})_{2}\right\}_{2} \cdot \mathrm{C}_{60}$ were obtained by evaporation of a solution containing fullerene acceptors and cadmium diethyldithiocarbamate donors by the method described in [18]. The XRD data show that the $\left\{\mathrm{Cd}(\mathrm{dedtc})_{2}\right\}_{2} \cdot \mathrm{C}_{60}$ complex acquires a monoclinic structure, space group $P 2_{1} / c$ with unit cell parameters $a=16.368(3), b=17.056(2)$, $c=10.6650(15) \AA, \beta=100.058(14)^{\circ}$ and $V=2931.63 \AA^{3}$ [7]. In the fullerene layers, each $\mathrm{C}_{60}$ molecule is surrounded by six neighbors in a nearly hexagonal manner with the shortest distances between the centers of the fullerene molecules being $10.058 \AA$ (four neighbors) and $10.665 \AA$ (two neighbors). No $\mathrm{C}-\mathrm{C}$ bonds were found between the fullerenes and, therefore, the fullerene molecules of the complex are monomeric at ambient conditions. The Raman spectra were recorded in the back-scattering geometry using a LabRam HR micro-Raman setup equipped with a Peltier cooled $\left(-70{ }^{\circ} \mathrm{C}\right) \mathrm{CCD}$ detector. The $514.5 \mathrm{~nm}$ laser line was used for Raman excitation and the activation of the phototransformation. The laser beam was focused on the sample by a 50 objective with a laser power of $\sim 200 \mu \mathrm{W}$, before the diamond-anvil cell (DAC). Measurements of the Raman spectra at high pressure were carried out using a Mao-Bell type DAC. The $4: 1$ methanol/ethanol mixture was used as pressure transmitting medium and the ruby fluorescence technique for pressure calibration [19]. 


\section{Results and discussion}

Figure 1 illustrates the peculiarities of the pressure-assisted photo-induced transformation in the $\left\{\mathrm{Cd}(\mathrm{dedtc})_{2}\right\}_{2} \cdot \mathrm{C}_{60}$ complex as evidenced in its Raman spectra and their pressure and exposure time dependence. The bottom inset shows the time evolution of the Raman spectra of the $\left\{\mathrm{Cd}(\mathrm{dedtc})_{2}\right\}_{2} \cdot \mathrm{C}_{60}$ complex recorded at pressures $1.63,4.37$ and $6.37 \mathrm{GPa}$ in the region of the PP-mode under fixed excitation conditions of $\lambda_{\text {exc }}=514.5 \mathrm{~nm}$, laser power $200 \mu \mathrm{W}$ and 50 objective. For each pressure, a different, fresh site of the sample was used to probe the spectra evolution with the illumination time. Raman spectra were recorded consecutively at $1.63 \mathrm{GPa}$ under continuous laser illumination for $1800 \mathrm{~s}$ and with $300 \mathrm{~s}$ exposure time for each spectrum. The spectra do not evolve with time regarding their structure and intensity distributions. The Raman spectra at $4.37 \mathrm{GPa}$, recorded under continuous laser illumination for $9900 \mathrm{~s}$ and $900 \mathrm{~s}$ exposure time for each spectrum, exhibit gradual time-dependent changes associated with the appearance of new bands shifted to lower energies with respect to the $\mathrm{A}_{g}(2)$ mode of the $\mathrm{C}_{60}$ monomer. The initial Raman spectrum, recorded with $900 \mathrm{~s}$ exposure time is similar to the Raman spectra at $1.63 \mathrm{GPa}$, while longer time laser illumination leads to a photoinduced transformation manifested by changes in the band structure and intensity distribution. Similar changes were also observed in a number of the degenerate $\mathrm{Hg}$ modes for the fullerene molecule. The Raman spectra at $6.37 \mathrm{GPa}$ were recorded under the same laser illumination conditions as the spectra at $4.37 \mathrm{GPa}$ but they do not show any time-dependent changes. The series of Raman measurements at various pressures have shown that the photo-induced transformation takes place in the pressure region $2.24-5.99 \mathrm{GPa}$. The relative intensities of the new bands increase with exposure time, indicating an increase in the photo-oligomers content in the fullerene layers, as in the case for photopolymerization in pristine $\mathrm{C}_{60}$ and $\left\{\mathrm{Pt}(\mathrm{dbdtc})_{2}\right\} \cdot \mathrm{C}_{60}$ complex at ambient conditions [14,20]. The ratio for the sum of the integrated intensities of the new PP-mode components to the total spectral intensity in the frequency region of the PP-mode provides a measure for the polymer content. The dependence of the photopolymer content on the laser exposure time is shown in Fig. 1 (circles) along with the fitting of the data by an exponential growth function (dotted line):

$$
P(t)=P_{\text {sat }}\{1-\exp (-t / \tau)\} .
$$

where $P(t)$ is the time-dependent content of the photopolymers, $P_{\text {sat }}$ is the content of the photopolymer attained after saturation of the phototransformation, and $\tau$ is the exponential growth time constant. The phototransformation parameters at $2.77 \mathrm{GPa}$ are $P_{\text {sat }}=64 \%$ and $\tau=889 \mathrm{~s}$. As shown earlier in the photopolymerization study of $\left\{\mathrm{Pt}(\mathrm{dbdtc})_{2}\right\} \cdot \mathrm{C}_{60}$ complex at ambient conditions [20], the parameter $P_{\text {sat }}$ increases, while the time constant $\tau$ decreases with an increase in the laser power. In fact, these parameters depend on the laser power density, which implies that it is necessary to carefully focus the laser beam during measurements. The top inset shows the dependence of the photopolymer content $P_{\text {sat }}$ on pressure. The Raman measurements in all pressure values were performed under the same conditions. The photopolymerization was initiated abruptly at $2.24 \mathrm{GPa}$ after the phase transition near $2 \mathrm{GPa}$ [9], occurs up to $5.99 \mathrm{GPa}$ and disappears at $6.37 \mathrm{GPa}$. The value of $P_{\text {sat }}$ varies between $60 \%$ and $80 \%$ despite the fixed illumination conditions, which may be attributed to somehow different focusing of the laser beam in different measurements.

Figure 2 illustrates the pressure dependence of the Raman bands of the $\left\{\mathrm{Cd}(\mathrm{dedtc})_{2}\right\}_{2} \cdot \mathrm{C}_{60}$ complex at pressures up to $6.5 \mathrm{GPa}$. The open circles mark the frequencies of the peaks related to the $\mathrm{H}_{g}(7)$ and $\mathrm{A}_{g}(2)$ modes recorded after short exposure time in the time-dependent set of Raman measurements, whereas the semi-filled circles correspond to the frequencies of the 


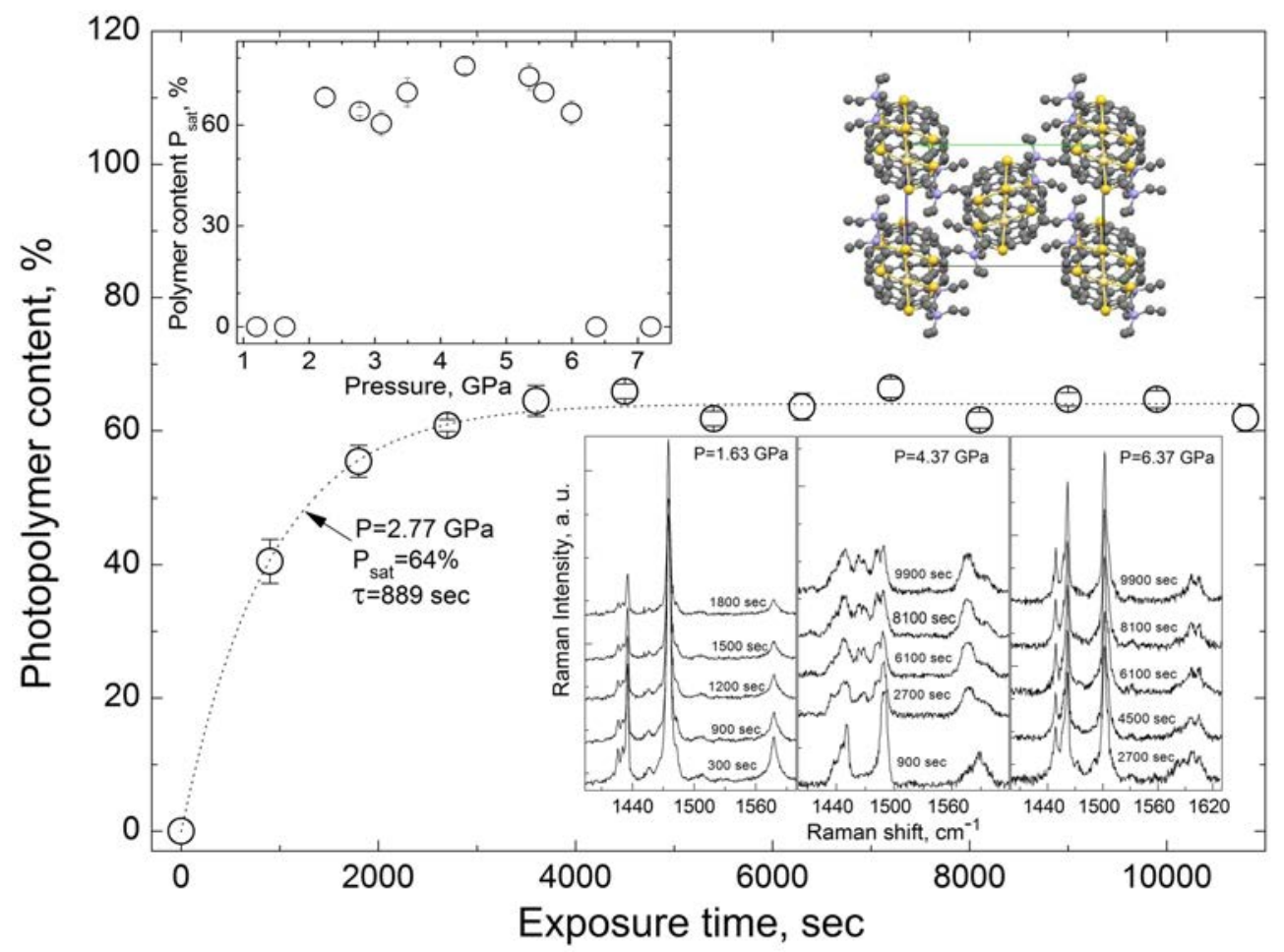

FIG. 1. Photopolymer content in the $\left\{\mathrm{Cd}(\mathrm{dedtc})_{2}\right\}_{2} \cdot \mathrm{C}_{60}$ complex at $2.77 \mathrm{GPa}$, excited with $200 \mu \mathrm{W}$ at $514.5 \mathrm{~nm}$, as a function of the illumination time and fitted by an exponential growth function (dotted line). The molecular arrangement in the fullerene complex viewed along the a-axis of the unit cell is also included. Bottom inset: Time evolution of the Raman spectra of the $\left\{\mathrm{Cd}(\mathrm{dedtc})_{2}\right\}_{2} \cdot \mathrm{C}_{60}$ complex in the region of the PP-mode at $1.63 \mathrm{GPa}$ (left), $4.37 \mathrm{GPa}$ (center) and $6.37 \mathrm{GPa}$ (right). Top inset: Pressure dependence of the saturated photopolymer content

split PP-mode components recorded after long exposure time when the photopolymer content is saturated. The solid lines are linear fits of the experimental data related to the $\mathrm{H}_{g}(7)$ and $\mathrm{A}_{g}(2)$ modes, while the dotted lines are fits of the split PP-mode components. Stars denote the frequencies of the split PP-mode components extrapolated to ambient pressure. The pressure evolution of the main PP-mode frequency exhibits an irregularity near $\sim 2 \mathrm{GPa}$ : the pressure coefficient between 2 and $3 \mathrm{GPa}$ is almost zero, whereas before and after this region it is $\sim 5 \mathrm{~cm}^{-1} / \mathrm{GPa}$. Thus, the Raman data recorded after short laser exposure time show the pressure-induced phase transition near $\sim 2 \mathrm{GPa}$ was most likely associated with covalent bonding between $\mathrm{C}_{60}$ molecules in fullerene layers. The phase transition was confirmed independently by XRD measurements which showed a distinct change in the material compressibility along the fullerene layers [9]. The intriguing result of the high pressure Raman study is that the pressure-induced phase transition activates the photo-induced transformation as it follows from the appearance of the split PP-mode components under prolonged laser illumination.

Figure 3 shows the frequencies of the $\mathrm{A}_{g}(2)$ PP-modes of various crystalline polymers of $\mathrm{C}_{60}$ (circles) vs. the number of the $s p^{3}$-like coordinated carbon atoms per fullerene molecular cage $[17,21]$. The Raman frequencies of the photo-oligomers in $\mathrm{C}_{60}$ fullerite (hexagons) are also included [20]. The structural arrangements of the intermolecular covalent bonds in different HPHT crystalline polymers, as deduced by detailed XRD measurements, are also illustrated in the insets $[12,13,22]$. The empirical dependence of the PP-mode frequencies of crystalline 


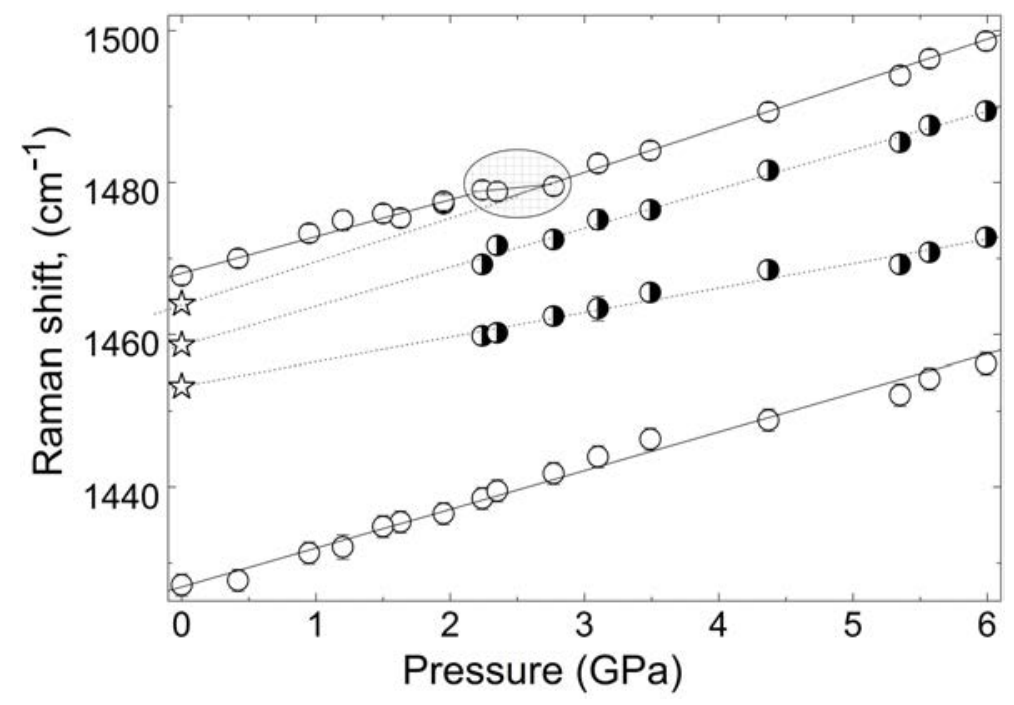

FIG. 2. Pressure dependence of the $\left\{\mathrm{Cd}(\mathrm{dedtc})_{2}\right\}_{2} \cdot \mathrm{C}_{60}$ complex Raman peaks related to the $\mathrm{H}_{g}(7)$ and $\mathrm{A}_{g}(2)$ modes of the $\mathrm{C}_{60}$ molecule at pressure up to 6.1 GPa. The open circles denote the peak frequencies recorded under short exposure time, while the semi-filled circles the frequencies of the split components of the PP-mode recorded under long exposure time. The lines are linear fits of the experimental pressure dependencies, while the stars show the extrapolated frequencies of the split PP-mode components to ambient pressure

$\mathrm{C}_{60}$ polymers and the photo-oligomers in $\mathrm{C}_{60}$ fullerite exhibits a monotonic decrease with an increase in the number of the $s p^{3}$-like coordinated carbon atoms per $\mathrm{C}_{60}$ molecule. Note, that the Raman band at $\sim 1452 \mathrm{~cm}^{-1}$ in phototransformed $\mathrm{C}_{60}$ is related to the conjugated linear chains that were first observed in the pressure-assisted photo-induced transformation of the linear orthorhombic $\mathrm{C}_{60}$ polymer [21-23]. The frequencies of the pressure-induced dimers, as well as the pressure-assisted photo-induced oligomers in the $\left\{\mathrm{Cd}(\mathrm{dedtc})_{2}\right\}_{2} \cdot \mathrm{C}_{60}$ complex extrapolated to ambient pressure are also shown in Fig. 3 (stars). These data agree well with those of the HPHT crystalline polymers and the photo-oligomers of $\mathrm{C}_{60}$ fullerite and may be attributed to dimers, fragments of linear chains and conjugated linear chains.

Summarizing, we have studied in detail the pressure-assisted photopolymerization of the fullerene molecules in the $\mathrm{C}_{60}$ layers of the molecular donor-acceptor complex $\left\{\mathrm{Cd}(\mathrm{dedtc})_{2}\right\}_{2} \cdot \mathrm{C}_{60}$, activated immediately after the pressure-induced phase transition near $2 \mathrm{GPa}$. The phototransformation process occurs at pressures ranging from $2.24-5.99 \mathrm{GPa}$. The photopolymerization rate and the saturated polymer content depend on the laser excitation wavelength, laser power and focusing but they are independent of pressure within this region. The photo-oligomers are, most likely, fragments of linear chains and conjugated linear chains whereas the pressureinduced phase transition results in the creation of $\mathrm{C}_{60}$ dimers. The photopolymerization of $\mathrm{C}_{60}$, which requires the rotation of the molecules in order to attain a suitable mutual orientation of the neighboring cages necessitated in the [2+2] cycloaddition reaction [14], may serve as the basis for the interpretation of the observed behavior. Then, the stability of the studied complex upon laser illumination at ambient and low pressure suggests that the molecular rotations are hindered. However, in the high pressure phase (above $2 \mathrm{GPa}$ ), the inter-cage distances are redistributed due to the pressure induced formation of dimers, leading to relatively large inter-dimer distances. This may allow some rotational degree of freedom that would explain the 


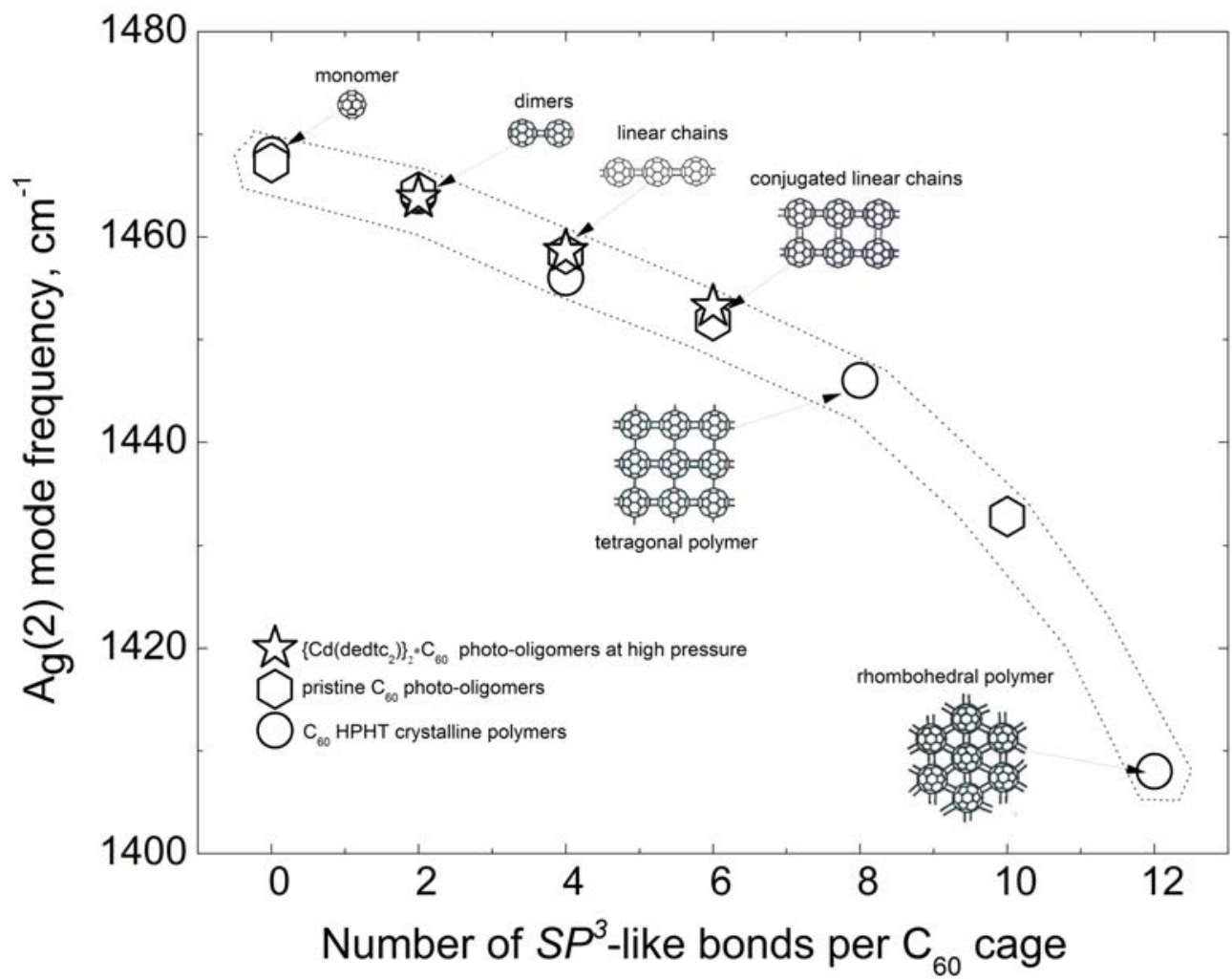

FIG. 3. The frequencies of the PP-modes of the HPHT crystalline polymers of $\mathrm{C}_{60}$ (circles), the photo-transformed $\mathrm{C}_{60}$ fullerite (hexagons) and the ambient pressure extrapolated frequencies of the photo-induced oligomers in $\left\{\mathrm{Cd}(\text { dedtc })_{2}\right\}_{2} \cdot \mathrm{C}_{60}$ complex at high pressure (stars) vs. the number of the $s p^{3}-$ like coordinated carbon atoms per $\mathrm{C}_{60}$ molecular cage. The arrangement of the intermolecular covalent bonds in HPHT crystalline polymers is also shown

photosensitivity of the material. Further volume reduction, for pressure above $6 \mathrm{GPa}$, freezes the rotations and renders the dimer phase immune to the laser irradiation.

\section{Acknowledgements}

The support by the Russian Foundation for Fundamental Research, grant No. 15-0201495, and the RAS Presidium Program "Physics of compressed matter" are greatly acknowledged. The authors are grateful to Dr. D. Konarev for providing the fullerene complexes.

\section{References}

[1] Konarev D.V., Khasanov S.S., et al. Formation of single-bonded $\left(\mathrm{C}_{60}^{-}\right)_{2}$ and $\left(\mathrm{C}_{70}^{-}\right)_{2}$ dimers in crystalline ionic complexes of fullerenes. J. Am. Chem. Soc., 2003, 125, P. 10074-10083.

[2] Konarev D.V., Khasanov S.S., et al. Negatively charged $\pi-\left(\mathrm{C}_{60}^{-}\right)_{2}$ dimer with biradical state at room temperature. J. Am. Chem. Soc., 2006, 128, P. 9292-9293.

[3] Meletov K.P., Dolganov V.K., Set al. Pressure-induced charge-transfer phase transition in crystalline $\mathrm{C}_{60} \cdot\left\{\mathrm{C}_{10} \mathrm{H}_{12} \mathrm{Se}_{42}\left(\mathrm{CS}_{2}\right)\right\}$ molecular complex studied by Raman spectroscopy. Chem. Phys. Lett., 1997, 281, P. 360-365.

[4] Cui Wen, Yao Mingguang, et al. Reversible polymerization in doped fullerides under pressure: the case of $\mathrm{C}_{60} \cdot\left\{\mathrm{Fe}\left(\mathrm{C}_{5} \mathrm{H}_{5}\right)_{2}\right\}_{2}$. J. Phys. Chem. B, 2012, 116, P. 2643-2650. 
[5] Meletov K.P., Konarev D.V. Raman study of the pressure-induced charge transfer transition in the neutral donor-acceptor complexes $\left\{\mathrm{Ni}\left(n \mathrm{Pr}_{2} \mathrm{dtc}\right)_{2}\right\} \cdot\left(\mathrm{C}_{60}\right)_{2}$ and $\left\{\mathrm{Cu}\left(n \operatorname{Pr}_{2} \mathrm{dtc}\right)_{2}\right\} \cdot\left(\mathrm{C}_{60}\right)_{2}$. Full. Nanotub. Carbon Nanostr., 2012, 20, P. 336-340.

[6] Meletov K.P., Konarev D.V. Raman study of the pressure-induced phase transitions in the molecular donoracceptor complex $\left\{\mathrm{Pt}(\mathrm{dbdtc})_{2}\right\} \mathrm{C}_{60}$. Chem. Phys. Lett., 2012, 553, P. 21-25.

[7] Meletov K.P. Phase transitions at high pressure in the donor-acceptor complexes of $\mathrm{C}_{60}$ studied by Raman spectroscopy. High Pressure Research, 2013, 33, P. 114-118.

[8] Meletov K.P. Phase transitions at high pressure in the fullerene $\mathrm{C}_{60}$ molecular donor-acceptor complex $\left\{\mathrm{Hg}(\text { dedtc })_{2}\right\}_{2} \cdot \mathrm{C}_{60}$. Physics Solid State, 2014, 56, P. 1689-1695.

[9] Meletov K.P., Konarev D.V., Tolstikova A.O. Phase transitions and photoinduced transformations at high pressure in the molecular donor-acceptor fullerene complex $\left\{\mathrm{Cd}(\mathrm{dedtc})_{2}\right\}_{2} \cdot \mathrm{C}_{60}$. JETP, 2015, 120, P. 989-997.

[10] Rao A.M., Zhou P., et al. Photoinduced polymerization of solid $C_{60}$ films. Science, 1993, 259, P. $955-957$.

[11] Stephens P.W., Bortel G., et al. Polymeric fullerene chains in $\mathrm{RbC}_{60}$ and $\mathrm{KC}_{60}$. Nature, 1994, 370, P. $636-639$.

[12] Iwasa Y., Arima T., et al. New phases of $\mathrm{C}_{60}$ synthesized at high pressure. Science, 1994, 264, P. $1570-1572$.

[13] Nunez-Regueiro M., Marques L., et al. Polymerized fullerite structures. Phys. Rev. Lett., 1995, 74, P. $278-281$.

[14] Zhou P., Dong Z.H., Rao A.M., Ecklund P.C. Reaction mechanism for photopolymerization of solid fullerene $\mathrm{C}_{60}$. Chem. Phys. Lett., 1993, 211, P. 337-340.

[15] Pusztai T., Oszlanyi G., et al. Bulk structure of phototransformed C $_{60}$. Solid State Commun., 1999, 111, P. 595-599.

[16] Karachevtsev V.A., Mateichenko P.V., et al. Effective photopolymerization of $\mathrm{C}_{60}$ films under simultaneous deposition and UV light irradiation: Spectroscopy and morphology study. Carbon, 2004, 42, P. 2091-2098.

[17] Davydov V.A., Kashevarova L.S., et al. Spectroscopic study of pressure polymerized phases of $\mathrm{C}_{60}$. Phys. Rev. B, 2000, 61, P. 11936-11945.

[18] Konarev D.V., Khasanov S.S., et al. Fullerene complexes with divalent metal dithiocarbamates: structures, magnetic properties, and photoconductivity. Russ. Chem. Bull. Int. Ed., 2007, 56, P. 2145-2161.

[19] Jayaraman A. Ultrahigh pressures. Rev. Sci. Instrum., 1986, 57, P. 1013-1031.

[20] Meletov K.P., Arvanitidis J., Christofilos D., Kourouklis G. Comparative Raman study of the photo-oligomers stability in the donor-acceptor fullerene complex $\left\{\mathrm{Pt}(\mathrm{dbdtc})_{2}\right\} \cdot \mathrm{C}_{60}$ and pristine $\mathrm{C}_{60}$. Nanosystems: Physics, Chemistry, Mathematics, 2016, 7 (1), P. 125-132.

[21] Meletov K.P., Davydov V.A., et al. High pressure photoinduced polymerization of the orthorhombic polymeric phase of $\mathrm{C}_{60}$. Chem. Phys. Lett., 2005, 416, P. 220-224.

[22] Le Parc R., Levelut C., et al. In situ X-ray powder diffraction study of the one-dimensional polymeric $\mathrm{C}_{60}$ phase transformation under high-pressure. Chem. Phys. Lett., 2007, 438, P. 63-66.

[23] Meletov K.P., Davydov V.A., et al. The influence of pressure on the photopolymerization rate of linear orthorhombic polymer of $\mathrm{C}_{60}$. Chem. Phys. Lett., 2006, 428, P. 298-302. 